\title{
Clinical features of 47 secondary hemophagocytic lymphohistiocytosis patients complicated with capillary leak syndrome
}

\author{
Changfeng Man ${ }^{1} \cdot$ Mengmeng Wang ${ }^{1}$. Guangli Yin ${ }^{1} \cdot$ Jiayu Huang ${ }^{1} \cdot$ Wanying Cheng ${ }^{1} \cdot$ Xing Wu $^{1} \cdot$ Lingling Liu $^{1}$. \\ Xin $\mathrm{GaO}^{2} \cdot$ Jujuan Wang ${ }^{2} \cdot \operatorname{Tian}_{\mathrm{Tian}^{2}}^{2} \cdot$ Limin Duan $^{2} \cdot \mathrm{Ji} \mathrm{Xu}^{2} \cdot$ Hongxia Qiu ${ }^{1,2} \mathbb{D}$
}

Received: 24 April 2020 / Revised: 6 September 2020 / Accepted: 16 September 2020 / Published online: 9 October 2020

(c) Japanese Society of Hematology 2020

\begin{abstract}
The clinical features of patients with secondary hemophagocytic lymphohistiocytosis (sHLH) complicated with capillary leak syndrome (CLS) remain controversial. The data of $259 \mathrm{sHLH}$ patients were retrospectively analyzed. The clinical manifestations, laboratory findings, treatment, and prognosis of the CLS-sHLH group and non-CLS-sHLH group were compared. The levels of fibrinogen, albumin, and serum calcium in the CLS-sHLH group were lower than in the non-CLS-sHLH group, and serum triglycerides in the CLS-sHLH group were higher than in the non-CLS-sHLH group $(P<0.05)$. Univariate analysis showed that fibrinogen level was an independent prognostic factor in sHLH patients complicated with CLS. The median survival time was significantly shorter in patients with fibrinogen $\leq 1.3 \mathrm{~g} / \mathrm{L}$ than in patients with fibrinogen $>1.3 \mathrm{~g} / \mathrm{L}$ $(P<0.05)$. Patients with improved CLS conditions in the CLS-sHLH group had significantly increased albumin and serum calcium after treatment $(P<0.05)$; patients without improved conditions in the CLS-sHLH group also had significantly increased albumin after treatment $(P<0.05)$, but the serum calcium did not change significantly $(P>0.05)$. sHLH patients complicated with CLS had significantly worse prognosis than without CLS. Significant reduction in fibrinogen may be an independent prognostic factor for poor prognosis in sHLH patients complicated with CLS.
\end{abstract}

Keywords Secondary hemophagocytic lymphohistiocytosis $\cdot$ Capillary leak syndrome $\cdot$ Risk factors $\cdot$ Prognosis

\section{Introduction}

Capillary leak syndrome (CLS) has varied clinical manifestations, such as generalized edema, weight gain, hypotension, hypoalbuminemia, oliguria, and polyserositis. CLS results from massive fluid and protein leakage due to an impaired capillary endothelium and increased vascular permeability caused by various disease conditions [1]. According to the etiology, CLS can be divided into idiopathic and secondary CLS [2]. Idiopathic CLS is caused by an unexplained episodic systemic capillary hyperpermeability. Secondary CLS is secondary to other, underlying diseases,

Hongxia Qiu

qiuhongxia@njmu.edu.cn

1 Department of Hematology, Jiangsu Province Hospital, The First Affiliated Hospital of Nanjing Medical University, 300 Guangzhou Road, Nanjing 210029, China

2 Department of Geriatric Hematology, Jiangsu Province Hospital, The First Affiliated Hospital of Nanjing Medical University, 300 Guangzhou Road, Nanjing 210029, China including hemophagocytic lymphohistiocytosis (HLH), and their treatment. HLH is triggered by excessive immune activation and excessive cytokine production by abnormally high numbers of lymphocytes and macrophages [3]. According to the etiology, HLH can be divided into primary HLH and secondary HLH (sHLH) [4]. sHLH is a disease with excessive immune activation caused by factors such as infection, tumors, and autoimmune diseases, usually without known genetic defects or family history [5].

The most common cause of secondary CLS is sepsis, followed by malignant hematological diseases such as lymphoma, acute leukemia, and HLH. Secondary CLS can also be caused by extracorporeal circulation treatment, severe trauma, implantation syndrome, differentiation syndrome, ovarian hyperstimulation syndrome, viral hemorrhagic fever, autoimmune diseases, snake bites, and ricin poisoning. Drugs that can be used for treating secondary CLS include interleukin (IL)-2, IL-11, some monoclonal antibodies, and gemcitabine [1, 6-9].

There are very few reports on CLS related to HLH, which may be related to the insufficient understanding of 
the disease by clinicians. By retrospectively analyzing the clinical features and laboratory data of $47 \mathrm{sHLH}$ patients complicated with CLS and 212 sHLH patients without CLS, this study aims to investigate the general clinical characteristics, treatment, and prognosis of sHLH patients complicated with CLS.

\section{Materials and methods}

\section{Study subjects}

The study subjects were 259 newly diagnosed sHLH patients in our hospital between January 2015 and December 2018. All diagnoses met the HLH-2004 diagnostic criteria. Based on whether they were complicated with CLS, the patients were divided into the CLS-sHLH group and non-CLS-sHLH group. There were 47 patients in the CLS-sHLH group, including 31 males and 16 females, with a median age of $50(13-85)$ years. There were 212 patients in the non-CLSsHLH group, including 119 males and 93 females, with a median age of 52 (15-86) years. The baseline clinical characteristics of patients in the two groups are shown in Table 1. The patients in the CLS-sHLH group were divided into the CLS remission subgroup and the CLS non-remission subgroup based on the disappearance of CLS symptoms after treatment. There were 35 and 12 patients in the CLS remission subgroup non-remission subgroup, respectively.

\section{Diagnostic criteria}

HLH diagnosis was based on the HLH-2004 diagnostic criteria [4]. HLH can be diagnosed if one of the following two conditions is met: (1) the presence of genetic defects
Table 1 Baseline clinical characteristics of CLS-sHLH group and non-CLS-sHLH group

\begin{tabular}{|c|c|c|c|}
\hline Characteristics & CLS-sHLH group $(N=47)$ & $\begin{array}{l}\text { non-CLS-sHLH group } \\
(N=212)\end{array}$ & $P$ value \\
\hline Median age (years) & $50(13-85)$ & $52(15-86)$ & 0.599 \\
\hline Gender & & & 0.217 \\
\hline Male & $31(66.0)$ & $119(51.4)$ & \\
\hline Female & $16(34.0)$ & $93(43.9)$ & \\
\hline Fever $^{\mathrm{a}}$ & $47(100.0)$ & $212(100.0)$ & 1.00 \\
\hline Splenomegaly & $40(85.1)$ & $177(83.5)$ & 0.786 \\
\hline $\mathrm{ANC}\left(\times 10^{9}\right)$ & $0.93(0-3.4)$ & $0.94(0-4.1)$ & 0.898 \\
\hline $\mathrm{HB}(\mathrm{g} / \mathrm{L})$ & $89(43-142)$ & $92(37-154)$ & 0.387 \\
\hline $\operatorname{PLT}\left(\times 10^{9}\right)$ & $52(7-65)$ & $53(5-87)$ & 0.393 \\
\hline $\mathrm{TG}(\mathrm{mmol} / \mathrm{L})$ & $3.17(0.96-14.6)$ & $3.02(0.42-9.44)$ & $<0.001 *$ \\
\hline Fibrinogen $(\mathrm{g} / \mathrm{L})$ & $1.30(0.24-3.93)$ & $1.49(0.20-6.89)$ & $0.001 *$ \\
\hline EBV-infection & & & 0.236 \\
\hline Positivity (\%) & $23(48.9)$ & $83(39.2)$ & \\
\hline Negativity (\%) & $24(51.1)$ & $125(58.9)$ & \\
\hline Unknown & 0 & $4(1.9)$ & \\
\hline Bone marrow hemophagocytosis & & & 0.261 \\
\hline Present $(\%)$ & $41(87.2)$ & $170(80.2)$ & \\
\hline Absent (\%) & $6(12.8)$ & $42(19.8)$ & \\
\hline Ferritin $(\mu \mathrm{g} / \mathrm{L})$ & $3494(926.7-15000)$ & $3035(139-15000)$ & 0.251 \\
\hline $\mathrm{sCD} 25^{\mathrm{b}}$ & & & 0.126 \\
\hline$\geq 2000 \mathrm{ng} / \mathrm{L}$ & 45 (95.7) & $187(88.2)$ & \\
\hline$<2000 \mathrm{ng} / \mathrm{L}$ & $2(4.3)$ & $25(11.8)$ & \\
\hline Albumin (g/L) & $22.3(16.8-25)$ & $39(16.9-49.8)$ & $<0.001 *$ \\
\hline Serum calcium (mmol/L) & $1.875(1.31-3.84)$ & $1.99(0.86-2.87)$ & 0.039* \\
\hline
\end{tabular}

Continuous variables are presented as median with interquartile range (IQR) and categorical variables are shown as frequency and percentage $(n, \%)$

$C L S$ capillary leak syndrome, $s H L H$ secondary hemophagocytic lymphohistiocytosis, $A N C$ absolute neutrophil count, $H B$ hemoglobin, $P L T$ platelet, $T G$ triglyceride, $s C D 25$ soluble interleukin-2 receptor

*Significantly different

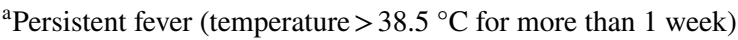

${ }^{\mathrm{b}}$ The serum level of sCD25 was measured by double antibody sandwich method with quantitative ELISA kit. The normal value range was $0-2000 \mathrm{ng} / \mathrm{L}$ 
of HLH confirmed by molecular biological examination; (2) five out of the following eight indicators are observed: fever (body temperature $>38.5^{\circ} \mathrm{C}$ ) lasting $>7$ days; splenomegaly; peripheral blood cell reduction by two or three criteria (hemoglobin $<90 \mathrm{~g} / \mathrm{L}$, platelets $<100 \times 10^{9} / \mathrm{L}$, absolute neutrophil count $\left.(\mathrm{ANC})<1.0 \times 10^{9} / \mathrm{L}\right)$ that was not caused by decreased bone marrow hematopoietic function; hypertriglyceridemia and/or hypofibrinogenemia (fasting triglycerides $>3 \mathrm{mmol} / \mathrm{L}$ or three standard deviations higher than the standard for the patient's age, fibrinogen $<1.5 \mathrm{~g} / \mathrm{L}$ or three standard deviations lower than the standard for the patient's age); phagocytosis in bone marrow, spleen, or lymph nodes; decreased or absent natural killer cell activity; serum ferritin $\geq 500 \mu \mathrm{g} / \mathrm{L}$; and increased soluble interleukin-2 receptor (sCD25) level.

Currently, there is no unified CLS diagnostic standard. The CLS diagnostic criteria in this study were the following [10]: after crystalloid fluid therapy, the blood pressure still progressively decreased, with CVP $<5 \mathrm{~cm} \mathrm{H}_{2} \mathrm{O}$ or lower than 90/60 mmHg; serum albumin $<25 \mathrm{~g} / \mathrm{L}$; progressive edema, weight gain, or accompanying polyserositis; reduced oxygenation index; chest X-ray showing pleural effusion; and critical illness after fluid resuscitation in patients who did not have edema caused by cardiogenic, hepatic nephrotic, neurogenic, hemorrhagic, or septic shock-related factors [6]. In this study, all 47 CLS-sHLH patients had CLS at the initial diagnosis of sHLH.

Patients in the CLS-sHLH group were divided into the CLS remission group and non-remission subgroup according to whether the symptoms of CLS disappeared or not. After treatment, the weight of patients in the CLS remission group decreased to before onset, edema and oliguria disappeared, blood pressure returned to normal, multi-serosal cavity effusion disappeared, and serum albumin and serum calcium increased significantly after treatment. The opposite was true for the group with no improvement in CLS.

\section{Clinical analysis experimental methods and follow-up}

The clinical and laboratory data of all sHLH patients complicated with CLS were retrospectively analyzed. All included patients underwent routine blood testing, a comprehensive metabolic panel, coagulation function testing, ferritin testing, virology [including Epstein-Barr virus (EBV), cytomegalovirus (CMV)], sCD25, blood and bone marrow culture, immunoglobulin, and rheumatoid panel testing; imaging (positron-emission tomography/computed tomography (CT) and/or CT, X-ray, B-mode ultrasound) examination; bone marrow smear and pathology; bone marrow cell chromosome analysis; and immunophenotyping.

The follow-up ended on February 15, 2019 and was mostly done through reviewing medical records and telephone calls. Overall survival (OS) is defined as the time from diagnosis to the end of the follow-up or the time of patient death.

\section{Statistical methods}

SPSS 24.0 software was used for statistical analysis. Measurement and count data were analyzed by the $t$ test and the Chi-squared test, respectively. Spearman correlation was used to analyze the correlation between variables. The Kaplan-Meier test and the log-rank method were used for survival analysis. $P<0.05$ was considered statistically significant.

\section{Results}

\section{Comparison of the basic characteristics between the two sHLH groups}

The CLS-sHLH group and non-CLS-sHLH group had 30 and 118 cases of lymphoma-associated hemophagocytic syndrome (LAHS) [11], respectively, which were not significantly different proportions $(P>0.05)$. Compared with the non-CLS-sHLH group, the CLS-sHLH group had lower levels of fibrinogen (FIB), albumin, and serum calcium and higher serum triglycerides $(P<0.05)$. The two groups had no significant differences in neutrophil count, hemoglobin, platelets, serum ferritin, or sCD25 $(P>0.05)$.

\section{Clinical characteristics of the 47 patients in the CLS-sHLH group}

All patients had varying degrees of bilateral pitting edema, which could be manifested as bilateral lower extremity edema, facial edema, and generalized edema; weight gain, with an average body weight increase of more than $10 \%$; progressively decreased blood pressure even after active crystalloid fluid therapy, with a blood pressure $<90 / 60 \mathrm{mmHg}$; average oxygen saturation $<95 \%$ and serum albumin $<25 \mathrm{~g} / \mathrm{L}$; and polyserositis observed by imaging study. Of them, 43 patients had oligouria symptoms $(<400 \mathrm{~mL} / 24 \mathrm{~h})$.

\section{The survival curves of the CLS-sHLH and non-CLS-sHLH groups}

Among the 47 patients in the CLS-sHLH group, 35 patients died, for a mortality rate of $74.5 \%$ (35/47). The deceased included 27 patients with malignant tumors, four with infection-related CLS-sHLH, two with autoimmune diseases, and two patients with CLS-sHLH of unknown cause. Among the 212 patients in the non-CLS-sHLH group, six were 
lost to follow-up, and 113 died. The mortality was $54.9 \%$ (113/206). There were 84 cases of malignant tumor-associated death, 26 cases of infection-related death, one case of autoimmune disease-related death, and two cases of death from unknown causes. The CLS-sHLH group and non-CLSsHLH group had a 1-year OS of $29.3 \%$ and $45.5 \%$, respectively $(P=0.048)$, and the 3 -year OS rates were $22.8 \%$ and $42.8 \%(P=0.014)$. The median survival time was 60 days in the CLS-sHLH group and 150 days in the non-CLS-sHLH group $(P=0.011)$. Figure 1 shows the survival curves of the CLS-sHLH group and the non-CLS-sHLH group.

\section{Subgroup analysis}

Age, sex, malignant tumor presence, and laboratory indicators with between-group differences (including fibrinogen, triglyceride, albumin, serum calcium ion) were included in Kaplan-Meier analysis to explore the prognostic factors for OS in the 47 patients in the CLS-sHLH group. The results showed that fibrinogen level was the only factor affecting the OS of sHLH patients complicated with CLS $(P<0.05)$ (Table 2). Fibrinogen $>1.3 \mathrm{~g} / \mathrm{L}$ vs $\leq 1.3 \mathrm{~g} / \mathrm{L}$ was used as the dichotomous variable to plot the survival curves. The curves showed that the median survival time of patients in the group with fibrinogen $\leq 1.3 \mathrm{~g} / \mathrm{L}$ was significantly shorter than that of the fibrinogen $>1.3 \mathrm{~g} / \mathrm{L}$ group (33 days vs 120 days, $P<0.05$ ). Figure 2 shows the comparison of OS between the sHLH-CLS patients in the fibrinogen $>1.3 \mathrm{~g} / \mathrm{L}$ group and the fibrinogen $\leq 1.3 \mathrm{~g} / \mathrm{L}$ group. The clinical symptoms of CLS in the CLS improvement subgroup (35 patients) disappeared after 5-14 days. Spearman correlation analysis showed that

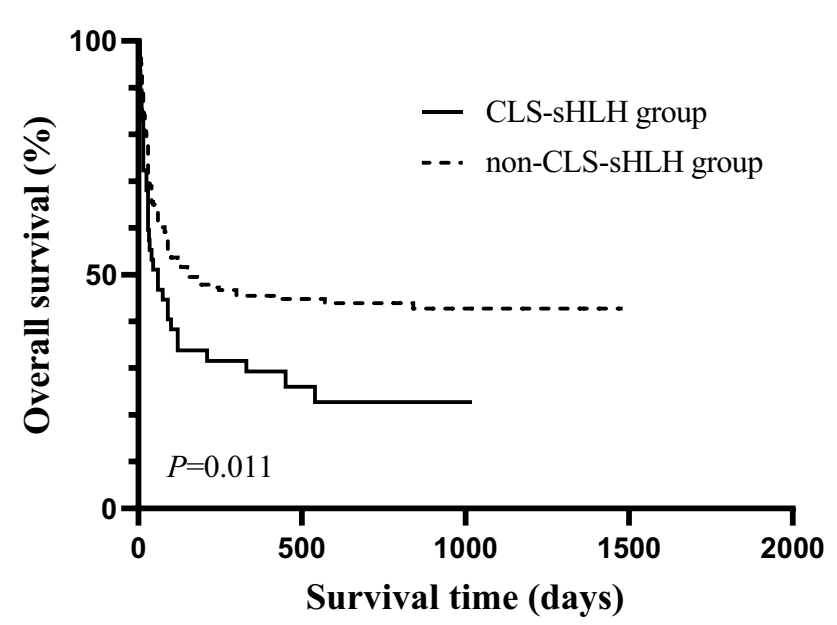

Fig. 1 The survival curves of the CLS-sHLH group and the nonCLS-sHLH group. Among the 47 patients in the CLS-sHLH group, 35 patients died; 212 patients in the non-CLS-sHLH group, six were lost to follow-up, and 113 died. The median survival time was 60 days in the CLS-sHLH group and 150 days in the non-CLS-sHLH group $(P=0.011)$
Table 2 Univariate analysis of prognostic factors affecting OS in patients with sHLH complicated with CLS

\begin{tabular}{lclll}
\hline Parameters & $N$ & $\begin{array}{l}\text { Median } \\
\text { OS (days) }\end{array}$ & $\chi^{2}$ & $P$ value \\
& & & 2.273 & 0.132 \\
\hline Age & 34 & 75 & & \\
$\quad<60$ years & 13 & 35 & & \\
$\quad \geq 60$ years & & & 0.18 & 0.671 \\
Gender & 31 & 60 & & \\
$\quad$ Male & 16 & 45 & & \\
$\quad$ Female & & & 4.227 & $\mathbf{0 . 0 4} *$ \\
Fibrinogen (g/L) & 24 & 33 & & \\
$\quad \leq 1.3$ & 23 & 120 & & \\
$\quad>1.3$ & & & 0.144 & 0.705 \\
$\quad$ Triglyceride (mmol/L) & 24 & 90 & & \\
$\quad<3.17$ & 23 & 30 & & \\
$\quad \geq 3.17$ & & & 0.003 & 0.96 \\
Albumin (g/L) & 24 & 45 & & \\
$\quad \leq 22.3$ & 23 & 60 & & \\
$\quad>22.3$ & & & 1.923 & 0.166 \\
$\quad$ Serum calcium (mmol/L) & 24 & 45 & & \\
$\quad \leq 1.875$ & 23 & 75 & & \\
$\quad>1.875$ & & & 1.989 & 0.158 \\
Underlying diseases & 15 & 90 & & \\
$\quad$ Non-malignancy-associated & & & \\
$\quad$ Malignancy-associated & 32 & 40 & & \\
\hline
\end{tabular}

*Significantly different

CLS improvement time was negatively correlated with survival time $(r=0.628, P<0.001)$.

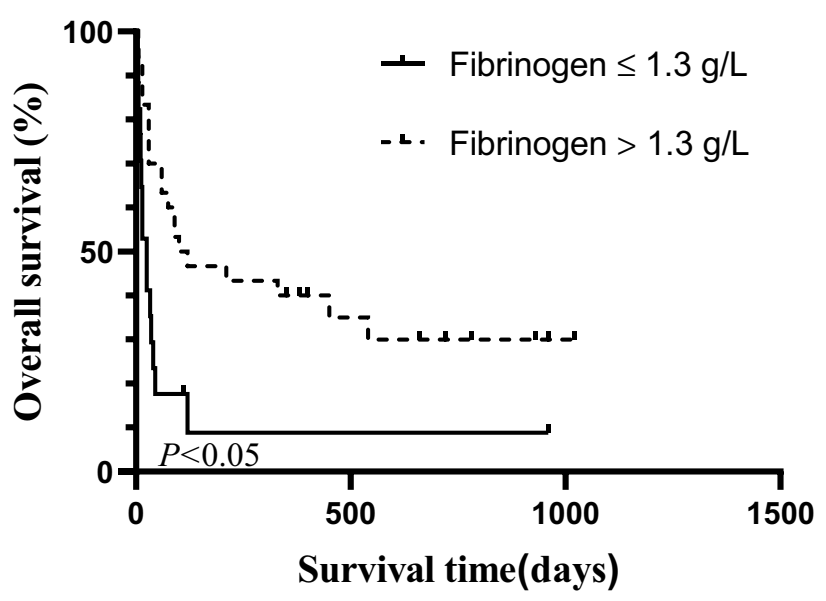

Fig. 2 The comparison of OS between the sHLH-CLS patients in the fibrinogen $>1.3 \mathrm{~g} / \mathrm{L}$ group and the fibrinogen $\leq 1.3 \mathrm{~g} / \mathrm{L}$ group. Fibrinogen $>1.3 \mathrm{~g} / \mathrm{L}$ vs $\leq 1.3 \mathrm{~g} / \mathrm{L}$ was used as the dichotomous variable to plot the survival curves. The curves showed that the median survival time of patients in the group with fibrinogen $\leq 1.3 \mathrm{~g} / \mathrm{L}$ was significantly shorter than that of the fibrinogen $>1.3 \mathrm{~g} / \mathrm{L}$ group (33 days vs 120 days, $P<0.05)$ 


\section{Therapeutic methods}

Treatment of the 47 patients in the CLS-sHLH group, for active treatment of sHLH, reasonable fluid therapy, diuretic, oxygen supply, and symptomatic supportive treatment were simultaneously provided. The sHLH treatment methods varied depending on etiology.

Treatment for the $32 \mathrm{sHLH}$ patients with malignant tumors, 17 patients complicated with LAHS were treated with dexamethasone combined with etoposide (VP-16) and/or liposomal doxorubicin. A lymphoma regimen was then applied after remission, four patients received a regimen of cyclophosphamide, epirubicin, oncovin, and prednisone (CEOP) or rituximab +CEOP; three received doxorubicin, etoposide, and prednisone (DEP); two received cyclophosphamide, doxorubicin, Oncovin, and prednisone; one patient (with Hodgkin's lymphoma) received adriamycin (doxorubicin), bleomycin, vinblastine, and dacarbazine chemotherapy; and three gave up treatment. Two patients with myelodysplastic syndrome also received DEP chemotherapy.

Treatment for ten patients with infection-related sHLH, eight patients were treated with hormones and anti-infective agents combined with immunoglobulin therapy, and VP-16 was not used. One patient with HIV was transferred to a local infectious disease hospital for anti-HIV treatment, and one patient with EBV was treated with HLH-1994 chemotherapy.

Treatment for three patients with autoimmune diseasesrelated HLH, all three patients were treated with immunosuppressive agents (cyclosporine, methotrexate, and/or cyclophosphamide) combined with hormone therapy. Two patients with sHLH of unknown cause were given HLH1994 chemotherapy.

In the CLS-sHLH group, 35 cases died eventually, among which eight cases died as follows: three cases abandoned treatment, three cases died due to cerebral hemorrhage (including two cases sudden loss of consciousness, one case seizures), one case suffered massive gastrointestinal hemorrhage, and one case died of acute pulmonary edema. Death occurred in 27 patients which were not sensitive or resistant to treatment, could aggravate CLS. Fifteen patients failed to accept systematic primary disease treatment after response, which resulted in HLH recurrence and death, including nine cases of lymphoma-associated HLH, five cases of infection-associated HLH, and one case of EBV-associated HLH. Twelve patients died of primary disease (lymphomaassociated HLH) progression, and there was no evidence of active HLH at death.

\section{Clinical features of patients in the CLS remission subgroup and the CLS non-remission subgroup}

The clinical symptoms of CLS disappeared in the 35 patients in the CLS remission subgroup after 5-14 days.
The CLS symptoms did not disappear in the 12 patients in the CLS non-remission subgroup, all of whom died within 15-30 days. After treatment, patients in the CLS remission subgroup had a body weight decrease back to the pretreatment weight, edema and oliguria disappeared, and blood pressure returned to normal. Polyserositis disappeared in 28 out of the 35 patients $(P<0.05)$. Serum albumin and serum calcium significantly increased and quickly reestablished after treatment $(P<0.05)$. After treatment, patients in the CLS non-remission subgroup still had hypotension, edema, oliguria, and polyserositis $(P>0.05)$, significantly increased albumin $(P<0.05)$, and no significant changes in serum calcium $(P>0.05)$. Prognostic factors for OS such as weight, edema, hypotension, oliguria, multiple serous effusions, albumin, and serum calcium.

\section{Discussion}

Currently, there are very few studies on sHLH-induced CLS, only a few case reports [12-14]. In clinical practice, we have found that HLH patients complicated with CLS had rapid disease progression and poor prognosis. Therefore, we explored the clinical features of HLH patients complicated with CLS to improve the clinical understanding of this disease.

This study included a total of 259 newly diagnosed sHLH patients in our hospital. Among them, 47 patients were complicated with CLS, for an incidence rate of $18.1 \%$, suggesting that the incidence of CLS is relatively high in sHLH patients. A large proportion of patients in this study had malignant lymphomas and EBV, and the rates of these were similar between the CLS-sHLH group and the nonCLS-sHLH group. sHLH has four causes: malignant tumors, infections, autoimmune diseases, and unknown causes. There were no significant differences in the distribution of causes of sHLH between the CLS-sHLH group and the nonCLS-sHLH group, suggesting that the cause of sHLH had no significant impact on the occurrence of CLS. Our results show that CLS was most common in patients with sepsis, but this does not suggest non-infection-related HLH patients are more prone to develop CLS.

The pathogenesis of sHLH-induced CLS is still not clear. One hypothesis is that excessive inflammatory cytokines due to HLH can damage the vascular endothelial cells, resulting in increased vascular permeability and the occurrence of CLS. Different degrees of pleural effusion lead to hypoxemia and hypoxia that can aggravate vascular endothelial cell injury, forming a vicious cycle and eventually leading to multiple-organ dysfunction syndrome (MODS) [15]. According to Tahirkheli et al. [16], CLS is often divided into a leakage period and a recovery period. Leakage period refers to the period of increased systemic capillary 
permeability, with clinical presentations such as progressive generalized edema, weight gain, hypoproteinemia, hypotension, and oliguria. In the recovery period, patients' capillary permeability gradually returns to normal, manifesting as gradual subsidence of edema, decreased body weight, increased blood pressure and central venous pressure, and increased urine output. Mullane et al. [17] reported that after active treatment, one patient had completely alleviated edema, reduced body weight, increased albumin, gradually disappearing symptoms, and continuously improving condition. In our study, all 47 patients in the CLS-sHLH group had clinical features of CLS at the early stage of sHLH. After active and effective treatment, the CLS symptoms disappeared in 5-14 days in the CLS improvement subgroup.

Druey et al. [18] found that CLS patients had hemoconcentration and hypoproteinemia, which easily led to venous thrombosis, resulting in coagulation factor deficiency. Cheng et al. [19] reported that CLS patients had decreased serum calcium. Xie et al. [20] showed that CLS patients had significantly higher-than-normal serum IL-1 $\beta$, IL-6, CCL2, VEGF, and CXCL10. However, there are very few studies on the changes in triglycerides and fibrinogen levels in patients with CLS. The present study found that fibrinogen, albumin, and serum calcium in the CLS-sHLH group were lower than in the non-CLS-sHLH group, and triglycerides in the CLS-sHLH group were higher than in the non-CLS-sHLH group, suggesting that the significantly decreased fibrinogen, albumin, and serum calcium and elevated triglycerides might indicate the possibility of CLS. In addition, this study found that albumin and serum calcium significantly increased in the CLS remission subgroup after active treatment (including albumin infusion). Albumin increased, but serum calcium did not, in the CLS non-remission subgroup, and these patients still had hypocalcemia. The causes of hypocalcemia in patients with sepsis may be impaired parathyroid function, inflammation cascade, hypomagnesemia, hypoalbuminemia, and increased procalcitonin. Chernow et al. [21], in a retrospective study, reported that in adult ICU patients, the incidence of hypocalcemia was closely related to the in-hospital mortality rate. Muller et al. [22] also showed that hypocalcemia affected more than two-thirds of critically ill patients and patients with sepsis, and hypocalcemia was associated with poor prognosis. Severe sHLH combined with CLS, excessive immune activation, and hypoalbuminemia result in lower serum calcium, which may adversely affect the prognosis. Our univariate analysis showed that fibrinogen level was an adverse prognostic factor affecting sHLH patients complicated with CLS. Low fibrinogen is one of the diagnostic criteria for HLH, and it is caused by factors such as abnormal coagulation function and impaired liver function. Signoff et al. [23] proposed that hypofibrinogenemia was associated with poor prognosis or sHLH in children with sepsis. When the hemophagocytic syndrome was complicated with CLS, the incidence of DIC was higher. Laboratory findings levels of inflammatory markers, including C-reactive protein, D-dimer, interleukin-6, interleukin-8, and TNF-alpha may suggesting DIC in patients with low level of fibrinogen.

sHLH patients complicated with CLS had rapid disease progression and critical condition. Once diagnosed, these patients need immediate treatment. HLH and CLS have similar clinical symptoms as many other diseases. In addition, due to an insufficient understanding of HLH and CLS, there are many missed diagnoses and misdiagnoses, delaying treatment, which might be one of the reasons for its high mortality. Currently, there is no specific treatment for CLS, and active treatment of primary disease based on fluid therapy is a key measure [15]. In this study, all CLS patients received active treatment for the primary diseases. Colloidal fluid (albumin, plasma, and hydroxyethyl starch) and crystalloid were properly provided, supplemented by diuretic and oxygen therapy.

Currently, there are two views on the application of albumin for CLS treatment. Wilkes and Navicick [24] found that albumin infusion not only did not significantly improve the survival rate of patients but also reduced the survival rate to some extent. During the leaking period of CLS, albumin can leak from capillaries and redistribute throughout the body after infusion, which has a relatively fast effect on colloid osmotic pressure. Currently, plasma substitutes, including macromolecules, are advocated for the treatment of CLS, while fresh-frozen plasma and human serum albumin are not recommended. Arif et al. [25] proposed that hypoproteinemia is a marker of acute respiratory distress syndrome in patients with severe pulmonary edema. Kawabe et al. [26] found that increasing the dose and rate of albumin infusion can play a protective role for patients with decreased serum total protein level, which can significantly improve their prognosis. Margarson et al. [27] proposed that hyperoncotic albumin infusion could increase interstitial fluid reabsorption and reduce capillary leakage. In the present study, symptoms of all included patients improved after albumin infusion combined with diuretic therapy. Even patients in the CLS non-remission subgroup had increased albumin after treatment, indicating that albumin infusion could effectively increase plasma colloid osmotic pressure and increase interstitial fluid reabsorption, showing a positive effect on volume expansion. After active treatment, 35 patients in this study had good results from their initial sHLH treatment and improved CLS. The initial treatment of sHLH in twelve patients failed, and CLS did not improve. Our findings confirm that timely and effective treatment of primary diseases could control disease progression. After the initial effective treatment of HLH, the symptoms can be completely alleviated and the control cytokine storm controlled, followed by 
improved CLS. If the cytokine storm cannot be effectively controlled by initial treatment, CLS will not be improved.

Lee et al. [15] found that when patients with sepsis developed CLS, the incidence of impaired endothelial cell function, shock, microvascular thrombosis, and organ failure increased, and thus the mortality rate significantly increased. Sauaia et al. [28] found that MODS score could reflect the extent of organ failure and predict mortality in critically ill patients. The MODS score increased in sHLH patients complicated with CLS. The occurrence of CLS may be related to the aggravation of HLH into the MODS stage. CLS can induce edema and dysfunction in vital organs and cells, thus aggravating the disease conditions. In the present study, the mortality in the CLS-sHLH group was higher than that in the non-CLS-sHLH group. The median survival time of the CLS-sHLH group was lower than that of the non-CLS-sHLH group, suggesting that the occurrence of CLS is a factor of poor prognosis in HLH patients. In addition, the correlation analysis showed that the longer the disease course of CLS was, the worse the prognosis was. Therefore, early diagnosis and early treatment are critical for sHLH patients with CLS.

\section{Conclusions}

The incidence of CLS is relatively high in sHLH patients (18.1\%). In sHLH patients, the occurrence of CLS has no significant correlation with the etiology of sHLH. Fibrinogen level may be a prognostic factor for sHLH patients with CLS (those with fibrinogen $\leq 1.3 \mathrm{~g} / \mathrm{L}$ had a worse prognosis). sHLH patients combined with CLS had rapid disease progression and poor prognosis. The median survival time was significantly lower in SHLH patients complicated with CLS than those without CLS. For patients with improved CLS, the longer the time required for CLS to improve, the worse the prognosis.

Acknowledgements The authors thank the participating patients, their families, and staff at the study site.

Author contributions CFM, MMW and HXQ designed the experiment. CFM and MMW performed the experiments. CFM, MMW, GLY, JYH, WYC, XW, XG, JJW, TT, LMD, JX and HXQ organized the clinical materials. CFM and MMW performed the data analysis. CFM wrote the paper. All authors contributed to the final approval of the manuscript.

Funding This work was supported by National Natural Science Foundation of China [grant number 81570175].

Data availability The data sets used and/or analyzed following standard procedures during the current study and are available from the corresponding author on reasonable request.

\section{Compliance with ethical standards}

Conflict of interest No potential conflict of interest was reported by the authors.

Ethical approval All procedures in studies were performed in accordance with the 1964 Helsinki Declaration and its later amendments or comparable ethical standards. Informed consent was obtained from all individual participants included in the study. This study was approved by the ethics committee of the First Affiliated Hospital of Nanjing Medical University (Reference Number: 2019-SR-446).

\section{References}

1. Siddall E, Khatri M, Radhakrishnan J. Capillary leak syndrome: etiologies, pathophysiology, and management. Kidney Int. 2017;92(1):37-46.

2. Duron L, Delestre F, Amoura Z, Arnaud L. Idiopathic and secondary capillary leak syndromes: a systematic review of the literature. Rev Med Interne. 2015;36(6):386-94.

3. Druey KM, Greipp PR. Narrative review: the systemic capillary leak syndrome. Ann Intern Med. 2010;153(2):90-8.

4. Henter JI, Horne A, Arico M, Egeler RM, Filipovich AH, Imashuku S, et al. HLH-2004: diagnostic and therapeutic guidelines for hemophagocytic lymphohistiocytosis. Pediatr Blood Cancer. 2007;48(2):124-31.

5. Al-Samkari H, Berliner N. Hemophagocytic lymphohistiocytosis. Ann Rev Pathol. 2018;13:27-49.

6. Stiller B, Sonntag J, Dahnert I, Alexi-Meskishvili V, Hetzer R, Fischer T, et al. Capillary leak syndrome in children who undergo cardiopulmonary bypass: clinical outcome in comparison with complement activation and $\mathrm{C} 1$ inhibitor. Intensive Care Med. 2001;27(1):193-200.

7. Ohta H, Yumara-Yagi K, Sakata N, Inoue M, Kawa-Ha K. Capillary leak syndrome in patients with hemophagocytic lymphohistiocytosis. Acta Paediatr. 1994;83(10):1113-4.

8. Bajwa R, Starr J, Daily K. Gemcitabine-induced chronic systemic capillary leak syndrome. BMJ Case Rep. 2017;2017: bcr2017221068. https://doi.org/10.1136/bcr-2017-221068

9. Anderson BJ, Peterson LL. Systemic capillary leak syndrome in a patient receiving adjuvant oxaliplatin for locally advanced colon cancer. J Oncol Pharm Pract. 2016;22(5):725-8.

10. Marx G, Vangerow B, Burczyk C, Gratz KF, Maassen N, Cobas Meyer M, et al. Evaluation of noninvasive determinants for capillary leakage syndrome in septic shock patients. Intensive Care Med. 2000;26(9):1252-8.

11. Han L, Li L, Wu J, Li X, Zhang L, Wang X, et al. Clinical features and treatment of natural killer/T cell lymphoma associated with hemophagocytic syndrome: comparison with other T cell lymphoma associated with hemophagocytic syndrome. Leuk Lymphoma. 2014;55(9):2048-55.

12. Alkhunaizi AM, Kabbani AH, ElTigani MA. Chronic idiopathic systemic capillary leak syndrome: a case report. Allergy Asthma Clin Immunol. 2019;15:34.

13. Xie Z, Ghosh CC, Patel R, Iwaki S, Gaskins D, Nelson C, et al. Vascular endothelial hyperpermeability induces the clinical symptoms of Clarkson disease (the systemic capillary leak syndrome). Blood. 2012;119(18):4321-32.

14. Morino M, Kumakura R, Miura N, Sasaki N. Decreased hepatic uptake of gallium-67 citrate in haemophagocytic syndrome occurring concomitantly with capillary leak syndrome. Pediatr Radiol. 2003;33(11):804-6. 
15. Lee WL, Slutsky AS. Sepsis and endothelial permeability. N Engl J Med. 2010;363(7):689-91.

16. Tahirkheli NK, Greipp PR. Treatment of the systemic capillary leak syndrome with terbutaline and theophylline. A case series. Ann Intern Med. 1999;130(11):905-9.

17. Mullane R, Langewisch E, Florescu M, Plumb T. Chronic systemic capillary leak syndrome treatment with intravenous immune globulin: case report and review of the literature. Clin Nephrol. 2019;91(1):59-63.

18. Druey KM, Parikh SM. Idiopathic systemic capillary leak syndrome (Clarkson disease). J Allergy Clin Immunol. 2017;140(3):663-70.

19. Cheng HW, James AF, Foster RR, Hancox JC, Bates DO. VEGF activates receptor-operated cation channels in human microvascular endothelial cells. Arterioscler Thromb Vasc Biol. 2006;26(8):1768-76.

20. Xie Z, Ghosh CC, Parikh SM, Druey KM. Mechanistic classification of the systemic capillary leak syndrome: Clarkson disease. Am J Respir Crit Care Med. 2014;189(9):1145-7.

21. Chernow B, Zaloga G, McFadden E, Clapper M, Kotler M, Barton $\mathrm{M}$, et al. Hypocalcemia in critically ill patients. Crit Care Med. 1982;10(12):848-51.

22. Muller B, Becker KL, Kranzlin M, Schachinger H, Huber PR, Nylen ES, et al. Disordered calcium homeostasis of sepsis: association with calcitonin precursors. Eur J Clin Investig. 2000;30(9):823-31.

23. Signoff JK, Fitzgerald JC, Teachey DT, Balamuth F, Weiss SL. Hypofibrinogenemia is associated with poor outcome and secondary hemophagocytic lymphohistiocytosis/macrophage activation syndrome in pediatric severe sepsis. Pediatr Crit Care Med. 2018;19(5):397-405.

24. Wilkes MM, Navickis RJ. Patient survival after human albumin administration. A meta-analysis of randomized, controlled trials. Ann Intern Med. 2001;135(3):149-64.

25. Arif SK, Verheij J, Groeneveld AB, Raijmakers PG. Hypoproteinemia as a marker of acute respiratory distress syndrome in critically ill patients with pulmonary edema. Intensive Care Med. 2002;28(3):310-7.

26. Kawabe S, Saeki T, Yamazaki H, Nagai M, Aoyagi R, Miyamura S. Systemic capillary leak syndrome. Intern Med. 2002;41(3):211-5.

27. Margarson MP, Soni NC. Changes in serum albumin concentration and volume expanding effects following a bolus of albumin 20\% in septic patients. Br J Anaesth. 2004;92(6):821-6.

28. Sauaia A, Moore EE, Johnson JL, Ciesla DJ, Biffl WL, Banerjee A. Validation of postinjury multiple organ failure scores. Shock. 2009;31(5):438-47.

Publisher's Note Springer Nature remains neutral with regard to jurisdictional claims in published maps and institutional affiliations. 